\title{
Autotransplante da Valva Pulmonar na Posição Aórtica (Cirurgia de Ross)
}

\author{
Fernando Amaral, Francisco F. Moreira N eto, Ricardo N. Sgarbieri \\ Ribeirão Preto, SP
}

"Can we improve a valve that has been molded by millions of years of flow and pressure in the cardiac chambers?" (Donald Ross 1995).

Essa pergunta, exprimindo um momento de reflexão pessoal após três décadas de experiência, reflete um dos fundamentos básicos que justifica o emprego dessa alternativa terapêutica, como iremos demonstrar.

A doença valvar aórtica isolada na criança é entidade relativamente freqüente ${ }^{1}$, predominantemente de natureza congênita e raramente de origem reumática. Seu espectro de apresentação clínica é amplo, representado num extremo por recém-nascidos sintomáticos nas primeiras horas de vida e, no outro, por adultos assintomáticos com sopro. Entre estas duas formas encontram-se casos de gravidade variáveis, sendo geralmente diagnosticados pelo achado de um sopro em crianças oligo ou assintomáticas. Na grande maioria dos pacientes, o quadro clínico predominante é de uma estenose aórtica valvar, devendo, entretanto, ser lembrada a possibilidade da presença de formas mais complexas de obstrução caracterizadas pela associação de lesões sub e supravalvares. Nas formas graves da doença valvar, os procedimentos rotineiramente utilizados para seu alívio (valvotomia com cateter-balão e comissurotomia cirúrgica) estão longe de promover a cura desses indivíduos, apesar da freqüente redução do gradiente ventrículo esquerdoaorta e diminuição da hipertrofia ventricular esquerda. Em virtude das lesões residuais uniformemente presentes após esses procedimentos, há necessidade de seguimento cuidadoso desses pacientes. Neste contexto, há freqüentemente muita discussão em relação à escolha do segundo tipo de procedimento, usualmente necessário a médio prazo em virtude da gravidade das lesões.

A experiência adquirida, principalmente nas últimas quatro décadas, em relação à esta entidade, revela que a busca de um substituto valvar ideal continua em franca discussão. Em virtude deste grau de dificuldade, tem-se como princípio terapêutico básico a tentativa de preservação da valva nativa através de procedimentos conservadores (plastias). A propósito, estudos recentes têm procurado de-

Hospital do Coração de Ribeirão Preto/Fundação Waldemar B. Pessoa Correspondência: Fernando Amaral - Av. Independência 1379 - 14025-390 Ribeirão Preto - SP

Recebido para publicação em 12/12/97

Aceito em 18/2/98 monstrar a viabilidade da plastia cirúrgica em função da morfologia valvar, preferencialmente, indicada nas valvas aórticas tricúspides ${ }^{2}$. Por outro lado, técnicas criativas têm procurado demonstrar a possibilidade de plastia valvar efetiva após valvoplastia com cateter-balão, independente da morfologia valvar, procurando, assim, manter a valva nativa por um período maior ${ }^{3,4}$. Este tipo de plastia conservadora tem sido também empregada com o uso de pericárdio bovino, além do emprego de técnicas especiais ${ }^{5}$. Entretanto, em virtude da gravidade da lesão, torna-se comumente necessária a substituição valvar, decisão esta que costuma envolver fatores variados. As próteses biológicas têm a vantagem de dispensar o uso de anticoagulantes, todavia, sua durabilidade é menor, levando à calcificação precoce e necessidade de retroca valvar o que, a longo prazo, inviabiliza seu uso em crianças. Por outro lado, as próteses mecânicas têm maior durabilidade, exigindo, entretanto, controle rigoroso da anticoagulação devido sua propriedade trombogênica. Este controle é sabidamente difícil de ser realizado com sucesso, constituindo-se em problema relevante nos ambulatórios de cardiologia pediátrica.

Em 1967, Ross ${ }^{6}$ descreveu a possibilidade de substituição da valva aórtica doente pela valva pulmonar do próprio paciente. Três décadas se passaram desde esse relato e os resultados tardios desse tipo de procedimento ${ }^{7-9}$ indicam ser esse, muito provavelmente, o método ideal para substituição da valva aórtica em crianças e jovens, tendo sido empregado tanto na doença aórtica isolada quanto nas situações em que aparece associada a outras cardiopatias ${ }^{10}$. O objetivo desse trabalho é apresentar uma revisão atualizada desta opção terapêutica, suas indicações e resultados, além de tentar promover sua discussão em nível nacional, visto seu emprego ser raramente relatado no nosso meio.

\section{Histórico}

O uso de homoenxertos valvares (valva de cadáver preservada em condições especiais) na posição aórtica revela-nos uma longa história, rica em detalhes, em que se sobressai a importância da investigação experimental associada à prática clínica. $O$ primeiro enxerto homólogo foi realizado por Murray ${ }^{11}$, usando valva aórtica humana fresca, seguido pela experiência de Beall e col ${ }^{12}$. Mantendo a valva aórtica in situ, esses enxertos eram inseridos na aorta descendente, após a artéria subclávia esquerda e podiam durar 
até seis anos. Apesar da experiência, já iniciada na época, com o emprego da "valva de bola", Duran e Cunning, em 1962, em Oxford, descreveram um método de implante do homoenxerto na posição subcoronária ${ }^{13}$. Esses autores se baseavam no fato de que, se os enxertos na aorta descendente podiam durar até seis anos, por que não deveriam durar para sempre, principalmente, considerando-se o uso de uma valva natural. Toda essa metodologia de remoção da valva de cadáver, sua moldagem e inserção subcoronária foi aplicada experimentalmente ${ }^{14} \mathrm{e}$, caracterizando o princípio de investigação clínico-experimental, essas informações foram transmitidas a Donald Ross que, ainda em 1962, inseriu pela primeira vez um homoenxerto aórtico subcoronário num paciente de 43 anos com estenose aórtica grave ${ }^{15}$. É interessante mencionar que essa tomada de decisão, marcante quando se contempla a história do tratamento da lesão aórtica valvar, não foi feita de maneira eletiva: durante a cirurgia no paciente citado, no qual se procedia uma descalcificação valvar, ocorreu destruição involuntária da valva, optando-se pelo implante temporário de um homoenxerto, até que uma valva de Starr pudesse ser importada. É importante lembrar que a disponibilidade imediata desse homoenxerto ocorreu em virtude de haver, nessa ocasião, um número considerável de valvas aórticas humanas congeladas e esterilizadas com óxido de etileno que estavam sendo usadas, experimentalmente, para implante na aorta descendente, sob a supervisão de Lord Brock. Devido à excelente evolução do paciente, decidiu-se que o homoenxerto deveria ser mantido ${ }^{16}$. Estava assim iniciada, de maneira bastante peculiar, uma nova era no tratamento da estenose aórtica. Trabalhos subseqüentes confirmaram a eficácia inicial dessa forma de tratamento ${ }^{17,18}$. Todavia, em 1966-67 foi demonstrado que esses pacientes apresentavam uma tendência ao aparecimento de insuficiência valvar. Verificou-se, a seguir, que essa complicação, geralmente ocorrendo após sete anos de cirurgia, era provavelmente relacionada à calcificação do enxerto e ao método de preparo da valva ${ }^{16}$. Em vista dessa ocorrência de degeneração precoce do homoenxerto e também baseado nos estudos experimentais de Lower em 1960, Ross, em 1967, trocou a valva aórtica doente pela valva pulmonar do próprio paciente, colocando um homoenxerto na posição pulmonar ${ }^{6}$. Duas características tornavam então essa técnica atraente: o design perfeito da valva pulmonar igual ao da valva aórtica e a possibilidade de se trabalhar com tecido vivo. No lugar da valva pulmonar foi inserido um enxerto aórtico homólogo, baseado na experiência, já em andamento na época, de correção de atresia da valva pulmonar através dessa técnica ${ }^{20}$. Apesar de Cunning e Duran, em 1964, terem iniciado um período de investigação com a valva de porco, Ross manteve sua linha de conduta pois não acreditava que essa valva pudesse ser funcionalmente melhor que a valva humana. O impacto causado pela introdução dessa técnica tão inovadora foi considerável e sua aceitação inicial esbarrava em dois pontos fundamentais: a cirurgia era considerada complexa, visto transformar um procedimento simples de correção univalvar em uma dupla troca, além de se criar uma doença na valva pulmonar. Estes dois fatores levaram a um ceticismo inicial, principalmente em relação à comunidade médica britânica e a repercussão desta não aceitação inicial na história da estenose aórtica congênita torna-se difícil de ser avaliada. Além disso, logo ficou evidenciado que havia uma curva de aprendizado com morbimortalidade expressiva ${ }^{21}$.

\section{Técnica cirúrgica}

Este aspecto encontra-se bem detalhado em várias publicações ${ }^{6,25,28,31,36}$. Através esternotomia mediana e após heparinização $(3 \mathrm{mg} / \mathrm{kg})$, procede-se a canulação do átrio direito e da aorta. Após iniciada a circulação extracorpórea (CEC), a aorta ascendente é clampeada junto ao tronco braquiocefálico e, posteriormente, aberta longitudinalmente na sua porção proximal em direção ao seio não coronário. Os orifícios coronários são canulados, separadamente, para permitir perfusão contínua e anterógrada com sangue do perfusato em hipotermia leve $\left(28 \mathrm{a} 32^{\circ} \mathrm{C}\right)$, outra opção sendo o emprego de cardioplegia cristalóide gelada. A dissecção da artéria pulmonar é iniciada, separando-a da aorta e os pontos de sangramento que surgem são cauterizados com bisturi elétrico em baixa corrente. A artéria pulmonar é primeiramente transeccionada distalmente junto à bifurcação $\mathrm{e}$ então dissecada de cima para baixo até que a musculatura do ventrículo direito (VD) seja visualizada na margem posterior da dissecção. Neste estágio, atenção deve ser tomada com o tronco da coronária esquerda. Após liberação da parede posterior, é realizada uma pequena incisão na parede anterior da via de saída do VD, logo abaixo dos seios da valva pulmonar que é continuada transversalmente, sempre abaixo dos seios valvares. Tomando-se o cuidado de evitar a lesão do $1^{\circ}$ ramo septal da artéria descendente anterior, a valva pulmonar é cuidadosamente enucleada da musculatura septal sem invadi-la profundamente e depositada numa cuba imersa em sangue. A valva aórtica é, então, retirada, como de rotina, a perfusão contínua é suspensa e uma dose de cardioplegia gelada é fornecida, associada à hipotermia tópica para a realização do auto-enxerto, que pode ser feita de duas maneiras distintas: free-hand ou root replacement. $\mathrm{Na} 1^{\mathrm{a}}$, o enxerto é inserido na raiz da aorta em posição subcoronária, com duas linhas de sutura após ressuspenção das comissuras. A linha de sutura inferior contém pontos separados seguindo uma linha horizontal no plano da margem inferior do anel aórtico. O enxerto é então recortado, preservando-se os pilares da valva aórtica e seus correspondentes seios para evitar a oclusão coronária. $\mathrm{O} 2^{\circ}$, plano de sutura contínua é feito para suturar o remanescente da parede da artéria pulmonar nas comissuras aórticas. A ressuspenção dos pilares comissurais é reforçada com a utilização de pontos separados de prolene 4-0 com pledgets. A aortorrafia é realizada de maneira usual, em dois planos de sutura contínua com fio de propileno 4-0 e o clamp aórtico é removido.

$\mathrm{Na}$ técnica de root replacement, a inserção do autoenxerto é feita em bloco, necessitando, portanto, da secção e reimplante dos óstios coronários. Esta técnica apresenta 
algumas vantagens: manutenção da arquitetura valvar pulmonar e possibilidade de ampliação do anel aórtico, além de ser o método ideal para tratamento de abscessos da raiz aórtica. A CEC é estabelecida da mesma forma, entretanto, a aortotomia deve ser feita transversalmente e, após retirada dos botões coronários (óstio coronário associado a uma porção da parede aórtica), a valva e toda a parede aórtica são removidas. O enxerto pulmonar é então suturado em posição, usando-se pontos separados de polipropileno 3-0 no nível do anel aórtico e os óstios coronários reimplantados com sutura contínua de polipropileno 6-0. Finalmente, a sutura do coto distal do enxerto com a aorta é feita continuamente com polipropileno 4-0. Em ambas as técnicas, após retirada do ar das câmaras esquerdas, o clamp aórtico é removido e o coração volta a ser perfundido continuamente.

A atenção agora é direcionada para a reconstrução da via de saída do VD, usualmente realizada através de um homoenxerto aórtico ou pulmonar, geralmente criopreservado. O leito muscular de onde foi removida a valva pulmonar é inspecionado para revisão da hemostasia. O homoenxerto é preparado deixando-se uma borda muscular de aproximadamente 2 a $3 \mathrm{~mm}$ para ser usada na linha de sutura e sendo orientado de maneira tal que ocorra um alinhamento das cúspides exatamente igual ao alinhamento da valva nativa. A sutura é iniciada pelo coto distal do homoenxerto com a artéria pulmonar, usando-se fio de polipropileno 4-0. A linha de sutura proximal, junto à via de saída do VD, é feita com sutura contínua de polipropileno 40 , tendo-se o cuidado de não lesar o $1^{\circ}$ ramo septal. O paciente é reaquecido a $37^{\circ} \mathrm{Ce}$, após saída da $\mathrm{CEC}$, recomenda-se a realização de um ecocardiograma transesofágico para confirmação da competência da neovalva aórtica.

\section{Resultados}

A efetividade a longo prazo da cirurgia de Ross pode ser avaliada, inicialmente, pelos resultados do próprio autor, visto ser dele a experiência mais longa (30 anos). É interessante mencionar que, até 1995, excluindo os casos pioneiros de Ross, menos de 500 pacientes tinham sido operados através dessa técnica, envolvendo menos de 100 cirurgiões no mundo ${ }^{22}$. Com os resultados favoráveis, progressivamente demonstrados, foi criada uma central de registros desse tipo de cirurgia ${ }^{23}$, permitindo uma melhor avaliação desses resultados.

Em 1988, relataram-se 241 pacientes operados ${ }^{24}$. O tempo mais longo de seguimento era de 18 anos, a mortalidade precoce era de $6,6 \%$ e a tardia de $1,7 \%$. Nesses pacientes, não houve ocorrência de tromboembolia e nenhum deles estava anticoagulado. Reoperação por insuficiência da neovalva aórtica foi necessária em 36 (14\%) casos e a incidência de eventos com 18 anos de seguimento foi de aproximadamente $50 \%$.

Nos EUA, a técnica foi introduzida por Elkins, em Oklahoma, a partir de 1986. Antes de se envolver nesta experiência, esse autor avaliou seus resultados com técnicas tradicionais de alívio cirúrgico ${ }^{25}$. Assim, verificou que, entre 121 pacientes operados após o $5^{\circ}$ ano de vida, usandose técnicas rotineiras, $55 \%$ estavam vivos ou sem necessidade de reoperação após 15 anos de seguimento, enquanto que nos casos operados, abaixo de cinco anos, essa incidência era de $13 \%$. Esses dados enfatizam um aspecto relevante e já discutido em relação à cirurgia valvar aórtica na criança ou adulto jovem: a possível necessidade de reintervenção no futuro e a documentação da idade precoce à primeira cirurgia, como fator de risco evolutivo desfavorável. As vantagens da cirurgia de Ross em relação à necessidade de uma segunda intervenção podem ser verificadas pela experiência desse autor: em 45 pacientes submetidos à troca valvar (mecânica ou biológica) até o ano de 1986, somente 49\% não foram a óbito ou não necessitaram retroca após seis anos de seguimento. Por outro lado, em 107 pacientes submetidos à cirurgia de Ross após 1986, esse índice foi de $89 \%$, destacando o bom resultado a médio prazo dessa opção cirúrgica, num estudo de comparação técnica realizado pelo mesmo cirurgião. Uma outra contribuição importante de Elkins foi a comparação do potencial de crescimento da neovalva aórtica ${ }^{26}$, verificada a partir do seguimento de 86 pacientes operados entre nove meses e 27 anos de idade. Nesse grupo, com sete anos de seguimento, a sobrevida atuarial foi de $96 \%$ e, em somente $8 \%$ dos casos, houve necessidade de reintervenção na neovalva aórtica ou na valva pulmonar autóloga. Recentemente, o crescimento da neovalva aórtica foi também verificado em estudo ecocardiográfico de adultos operados ${ }^{27}$. Nesse trabalho, foi constatado rápido crescimento do enxerto durante os 10 primeiros dias do implante e crescimento subseqüente mais lento, sem ocasionar insuficiência aórtica importante no seguimento a médio prazo.

Um detalhe interessante, particularmente quando consideramos a possibilidade de realização da cirurgia no nosso meio, éo método ideal para substituição da valva pulmonar transplantada. Ross, no início de sua experiência ${ }^{24}$, usava enxerto homólogo aórtico, tendo optado posteriormente pelo enxerto homólogo pulmonar, conduta essa compartilhada por Elkins ${ }^{25}$. Estima-se que $80 \%$ desses enxertos pulmonares estariam funcionalmente adequados após 20 anos de seguimento ${ }^{26}$. A disponibilidade de enxerto homólogo no nosso meio é bastante recente e, apesar de algumas discussões legais, abre uma perspectiva bastante favorável. Uma alternativa é o emprego de tubo de pericárdio bovino mantido sem suporte, já empregado ${ }^{28}$ e considerado, teóricamente adequado nessas circunstâncias (Ronald Elkins, comunicação pessoal). É importante enfatizar que a possível necessidade de retroca devido à degeneração desses enxertos pode ser realizada, usualmente com baixa morbimortalidade.

Experiências de outros grupos, embora envolvendo menor número de pacientes ou com tempo menor de seguimento, são também bastante favoráveis ${ }^{29-31}$, algumas delas inclusive propondo modificações técnicas interessantes ${ }^{31}$. Basicamente, a experiência desses autores, apesar de limitada, revela baixo índice de complicações a curto prazo e re- 
sultados favoráveis, considerando o procedimento como ideal para troca valvar em crianças e adultos jovens. As formas mais graves de obstrução (complexas) podem ser tratadas através da associação de técnicas como a recentemente proposta aortoventriculoplastia (cirurgia de Ross-Kono) ${ }^{32}$, havendo inclusive relato de sua realização em neonatos com fibroelastose endocárdica ${ }^{33}$.

Uma experiênciainteressante é a relatada por Kouchoukos e $\operatorname{col}^{29}: 33$ pacientes operados entre 1989-93 (implantação da valva e tronco pulmonar como unidade): usando ecocardiograma intra-operatório para medir diâmetros valvar aórtico e pulmonar e também no pós-operatório, para avaliar a função da neovalva aórtica, esses autores relataram ausência de mortalidade hospitalar, enfatizando a segurança da técnica. Além disso, no seguimento a curto prazo, notouse crescimento uniforme da neovalva aórtica, ausência de dilatação progressiva do anel e ausência de piora da regurgitação aórtica, leve na maioria dos casos. Esses dados são bastantes semelhantes aos relatados por Sievers e col ${ }^{34}$.

\section{Comentários - experiência nacional}

A nosso ver, torna-se evidente que, baseado nas diversas experiências acima relatadas, a cirurgia de Ross parece ser, no momento, o método ideal quando há necessidade de troca valvar aórtica na criança e no adulto jovem. Em virtude de ser tecnicamente exigente, a experiência individual do cirurgião envolvido é fundamental para a adoção da técnica pelo grupo. A observância de detalhes técnicos, como a necessidade de perfeita coaptação dos folhetos, parece ser fator fundamental para o sucesso da cirurgia a longo prazo. Variações técnicas, como o implante da valva pulmonar e parte do tronco pulmonar como unidade pode, se demonstrado, constituir-se num outro dado a influenciar o resultado tardio. Um outro aspecto de fundamental importância é o diâmetro aumentado do anel aórtico em relação ao anel pulmonar, outrora considerado como contra-indicação para a cirurgia de Ross. Entretanto, estudos recentes, refletindo avanço técnico considerável no estudo da técnica, têm demonstrado a possibilidade do emprego de recursos técnicos criativos que diminuem esse diâmetro, mantendo o potencial de crescimento ${ }^{35}$.

A experiência nacional publicada é, curiosamente, pobre: a indisponibilidade do enxerto homólogo para reconstrução da via de saída do VD era, até recentemente, um problema no nosso meio, particularmente em crianças. Entretanto, essa dificuldade legal, aparentemente solucionada, não deve inviabilizar a curto prazo a realização do procedimento naqueles casos ainda pendentes: a válvula de porco montada sem suporte pode ser uma alternativa segura (Elkins, comunicação pessoal) e, caso ocorra degeneração (o que é esperado), a retroca valvar não deverá proporcionar aumento da mortalidade ${ }^{25,28}$. Furtado e col ${ }^{28}$ empregaram pela primeira vez a técnica no nosso meio. Usando tubo valvado de pericárdio bovino para reconstruir a via de saída do VD, os autores obtiveram bom resultado a curto prazo em paciente de 38 anos. Recentemente, Costa e $\operatorname{col}^{36}$, num trabalho pioneiro e de especial relevância no nosso meio, relataram sua experiência com 24 casos. Experiências interessantes e recentes da cirurgia de Ross realizada em neonatos e lactentes ${ }^{37,38}$, possibilidade de associação de técnicas em jovens com formas obstrutivas complexas ${ }^{39}$, assim como o uso recente da técnica em pacientes com tumores ${ }^{40}$, abrem uma perspectiva mais ampla para a aplicação desta técnica. Acreditamos que esses dados sejam suficientes para que outros serviços renomados adotem, criteriosamente, a técnica descrita por Ross, como já vem ocorrendo $^{41}$. Certamente que seria interessante a realização de um estudo prospectivo multicêntrico nacional para se avaliar a efetividade do procedimento no nosso meio.

\section{Conclusões}

A comparação de alternativas cirúrgicas é sempre difícil, devido a fatores como experiência e habilidade do cirurgião, critérios de indicação cirúrgica e infraestrutura local. Particularmente em experiências longas, as modificações técnicas e de cuidados operatórios emergentes durante o período tendem a influenciar substancialmente os resultados ao longo do tempo. Entretanto, é interessante mencionar os resultados de experiências com outras próteses. Recentemente, o Edinburgh Heart Valve Trial ${ }^{42}$ definiu o resultado tardio das trocas de valva aórtica, usando-se valvas de porco tipo Hancock e Carpentier-Edwards. É importante notar que nesse estudo, em pacientes menores de 50 anos de idade (teoricamente comparáveis com a série original de Ross), a ausência de reoperação em 12 anos foi somente $40 \%$, contrastanto com a cirurgia de Ross $(76 \% \mathrm{em}$ 10 anos). Um outro tipo de prótese, o enxerto homólogo aórtico, como já relatado ${ }^{43}$, apresentou ausência de reoperação em somente 54\% com 14 anos de seguimento.

Acreditamos que fatos como a adoção da técnica de Ross por outros grupos, assim como a demonstração de seus resultados iniciais favoráveis ${ }^{29,34}$, inclusive no nosso meio ${ }^{36}$, revelam a credibilidade da técnica, sendo, obviamente, fundamental a análise criteriosa dos resultados tardios para determinação de sua real eficácia. A experiência inovadora de Elkins nos EUA, já com resultados otimistas a médio prazo, constitui-se num marco importante para a sedimentação da cirurgia de Ross. Estudos recentes da função ventricular e, também, da evolução clínica de crianças operadas com essa técnica têm apresentado pacientes assintomáticos e com função ventricular esquerda adequada pelo ecocardiograma ${ }^{44}$. Essas informações, apesar do tempo de seguimento de três anos, são otimistas em relação ao prognóstico dessas crianças. Finalmente, os resultados a longo prazo, recentemente publicados ${ }^{45}$, são relevantes: 131 sobreviventes da série original de Ross foram estudados com tempo médio de seguimento de 21 anos: com 10 e 20 anos de seguimento tivemos, respectivamente: sobrevida dos pacientes ( $85 \%$ e $60 \%$ ), sobrevida do auto-enxerto pulmonar $(88 \%$ e $75 \%)$, sobrevida do enxerto homólogo na posição pulmonar (85\% e $79 \%$ ) e ausência de reoperação (73\% e $61 \%)$. Dos 53 óbitos tardios, 21 foram devidos à insuficiên- 
cia cardíaca. Entre 46 casos reoperados, 20 foram devido à insuficiência do auto-enxerto e 10 devido a estenose pulmonar. É interessante mencionar que células vivas foram encontradas no auto-enxerto até 24 anos após a cirurgia, permitindo-se inferir que esse enxerto deva durar para sempre. Entre os 72 sobreviventes até $1994,75 \%$ estavam na classe I da NYHA e $80 \%$ preservavam o auto-enxerto. Outra informação bastante relevante, também recentemente publicada, foi o relato da possibilidade de gestação bem sucedida nesses pacientes. Entre 27 pacientes operadas, assintomáticas e sem uso de anticoagulantes, foram registradas 14 gestações em oito delas. Não houve ocorrência de morte materna, acidente tromboembólico, hemorragia ou piora da função valvar durante a gravidez ${ }^{46}$. Estes dados são bastante especiais e revelam os resultados tardios mais atualizados que se tem notícia sobre este procedimento inovador. Espera-se que o seguimento adequado a longo prazo desses pacientes venha demonstrar uma possível solução definitiva para o difícil problema, freqüente na prática clínica, da criança ou jovem com doença valvar aórtica grave.

\section{Referências}

1. Kitchner DJ, Jackson M, Walsh K, Peart I, Arnold R - Incidence and Prognosis of congenital aortic valve stenosis in Liverpool (1960-1990). Br. Heart J 1993; 69: 71-9.

2. van Son JAM, Reddy VM, Black MD, Rajasinghe H, Haas GS, Hanley FL- Morphologic determinants favoring surgical aortic valvuloplasty versus pulmonary autograft valve replacement in children. J Thorac Cardiovasc Surg 1996; 111 1149-57.

3. Hawkins JA, Minich LL, Shaddy RE et al - Aortic valve repair and replacement after balloon aortic valvuloplasty in children. Ann Thorac Surg 1996; 61: 1355-

4. Lamberti JJ - The aortic valve: to dilate, repair, or replace - that is the question Ann Thorac Surg 1996; 61: 1297-8.

5. Tolan MJ, Daubeney PE, Slavik Z, Keeton BR, Salmon AP, Monro JL - Aortic valve repair of congenital stenosis with bovine pericardium. Ann Thorac Surg 1997; 63: 465-9.

6. Ross DN - Replacement of aortic and mitral valves with a pulmonary autograft. Lancet 1967; 2: 956-8.

7. Ross DN, Jackson M, Davies J - Pulmonary autograft aortic valve replacement: long-term results. J Cardiovas Surg 1991; 6(suppl): 529-33.

8. Elkins RC, Santangelo KL, Randolph JD et al - Pulmonary autograft replacement in children: the ideal solution? Ann Thorac Surg 1992; 216: 363-71.

9. Halees Z, Duran CMG - Pulmonary autograft for aortic valve replacement in rheumatic disease. An ideal solution? J Heart Valve Dis 1994; 3: 384-7.

10. Amaral FTV, Granzotti JA, Nunes MA, Sgarbieri RN, Somerville J - Seguimento tardio após correção de coarctação da aorta. Reintervenção cirúrgica subsequiente. Arq Bras Cardiol 1993; 61: 273-8.

11. Murray G-Homologous aortic valve segment transplants as surgical treatment for aortic and mitral insufficiency. Angiology 1956; 7: 446-51.

12. Beall AC, Morris GC, Cooley DA, DeBakey ME - Homotransplantation of the aortic valve. Thorac Cardiovasc Surg 1961; 42: 497-506.

13. Duran CG, Gunning AJ - A method for placing a total homologous aortic valve in the subcoronary position. Lancet 1962; ii: 488-9.

14. Cam CR, Aram HH, Mennell ER - An experimental study of aortic valve homografts. Surg Gynecol Obstetr 1952; 94: 129-31.

15. Ross DN - Homograft replacement of the aortic valve. Lancet 1962; $2: 487$.

16. Ross DN - Evolution of the homograft valve. Ann Thorac Surg 1995; 59: 565-7.

17. Davies H, Missen GAK, Blandford G, Roberts T, Lessof MH, Ross DN - Homograft replacement of the aortic valve: a clinical and pathological study. Am J Cardiol 1968; 22: 195-202.

18. Barratt-Boyes BG - Homograft aortic valve replacement in aortic incompetence and stenosis. Thorax 1964; 19: 131-50.

19. Lower R, Shafer RC, Shumway NE-Autotransplantation of the pulmonic valve into the aorta. J Thorac Cardiovasc Surg 1960; 39: 680-7.

20. Ross DN, Somerville J-Correction of pulmonary atresia with a homograft valve. Lancet 1966; 2: 1446-7.

21. Robles A, Vaugham M, Lau JK, Bodnar E, Ross DN - Long-term assessment of aortic valve replacement with autologous pulmonary valve. Ann Thorac Surg 1985; 39: 238-42

22. Westaby S - Pulmonary autograft replacement of the aortic valve. Br Heart J 1995; 74: 1-3.

23. Oury JH, Eddy AC, Cleveland JC - The Ross procedure: a progress report. J Heart Valve Dis 1994; 3: 361-4.

24. Matsuki O, Okita Y, Almeida RS et al - Two decades' experience with aortic valve replacement with pulmonary autograft. J Thorac Cardiovasc Surg 1988; 95: 705 11.

25. Elkins RC-Congenital aortic valve disease: evolving management. Ann Thorac Surg 1995; 59: 269-74
26. Elkins RC, Knott-Craig CJ, Ward KE, McCue C, Lane MM-Pulmonary autograft in children: realized growth potential. Ann Thorac Surg 1994; 57: 1387-94.

27. Hokken RB, Bogers AJJC, Meindert AT et al - Does the pulmonary autograft in the aortic position in adults increase in diameter? An echocardiographic study. J Thorac Cardiovasc Surg 1997; 113: 667-74

28. Furtado HB, Duran CA, Mejias GH et al - Transposição da valva pulmonar para substituição da valva aórtica. Rev Bras Cir Cardiovasc 1990; 5: 160-7.

29. Kouchoukos NT, Dávilla-Román VG, Spray TL, Murphy SF, Perrillo JB - Replacement of the aortic root with a pulmonary autograft in children and young adults with aortic valve disease. N Engl J Med 1994; 330: 1-6.

30. Schoof PH, Goome-Dijkuis AH, Bogers AJJC et al - Aortic root replacement with pulmonary autograft in children. J Thorac Cardiovasc Surg 1994; 107: 367-73.

31. Pacífico AD, Kirklin JK, McGiffin DC, Matter CJ, Narda DC, Deithelm AG - The Ross operation - early echocardiographic comparison of different operative techniques. J Heart Valve Dis 1994; 3: 365-70.

32. Reddy VM, Rajasinghe HA, Teitel DF, Haas GS, Hanley FL Aortoventriculoplasty with the pulmonary autograft. The "Ross-Konno" procedure. J Thorac Cardiovasc Surg 1996; 111: 158-67.

33. van Son JAM, Falk V, Mohr FW - Ross-Konno operation with resection of endocardial fibroelatosis for critical aortic stenosis with borderline-sized left ventricle in neonates. Ann Thorac Surg 1997; 63: 112-6.

34. Sievers HH, Leyh R, Loose R, Guha M, Petry A, Bernhard A - Time course of dimension and function of the autologous pulmonary root in the aortic position. $\mathrm{J}$ Thorac Cardiovasc Surg 1993; 105: 775-80.

35. Durham LA, des Jardins SE, Mosca RS, Bove EL - Ross procedure with aortic root tailoring for aortic valve replacement in the pediatric population. Ann Thorac Surg 1997; 64: 482-6.

36. CostaFDA, Poffo R, Gaspar Ret al-Experiência cirúrgica inicial com a operação de Ross (auto-enxerto pulmonar). Rev Bras Cir Cardiovasc 1996; 11: 86-95.

37. Runliman M, Ostman-Smith I, Westaby S - Infant Ross procedure for recurrent aortic stenosis. Heart 1996; 76 : 449-50.

38. Sudow G, Solymar L, Berggren H, Erikson B, Holmgren D, Gilljam T - Aortic valve replacement with a pulmonary autograft in infants with critical aortic stenosis. J Thorac Cardiovasc Surg 1996; 112: 433-6.

39. Starnes VA, Luciani GB, Wells WJ, Allen RB, Lewis AB - Aortic root replacement with the pulmonary autograft in children with complex left heart obstruction. Ann Thorac Surg 1996; 62: 442-9.

40. Giamberti A, Giannico S, Squitieri C et al - Neonatal pulmonary autograft implantation for cardiac tumors involving the aortic valve. Ann Thorac Surg 1995; 59: 1219-21.

41. Santini F, Stavri G, Edwards S, Dyke C, Yacoub M-Early results of a prospective randomized trial of homograft versus pulmonary autograft replacement of the aortic valve. Heart 1996; (suppl 1): 46.

42. Bloomfield P, Wheatley DJ, PrescottRJ, Miller HC - Twelve year comparison of a Bjork-Shiley mechanical heart valve with porcine bioprostheses. N Engl J Med 1991; 324: 573-9.

43. Barratt-Boyes BG, Roche AHG, Subramanyan R, Pemberton JR, Whitlock RMLLong-term follow-up of patients with the antibiotic-sterilized aortic homograft valve inserted freehand into the aortic position. Circulation 1987; 75: 768-77.

44. Hokken RB, Cromme-Dijkhuis AH, Bogers AJJ et al - Clinical outcome and left ventricular function after pulmonary autograft implantation in children. Ann Thorac Surg 1997; 63: 1713-7.

45. Chambers JC, Somerville J, Stone S, Ross DN-Pulmonary autograft procedure for aortic valve disease. Long-term results of the pioneer series. Circulation 1997; 96: 2206-14.

46. Dore A, Somerville J - Pregnancy in patients with pulmonary autograft valve replacement. Eur Heart J 1997; 18: 1659-62. 